\title{
Time development of the upper cloud edge in one-dimensional approximation based on moist thermodynamics
}

\author{
P. B. Rutkevich ${ }^{1}$, B. P. Rutkevych ${ }^{2}$, and G. S. Golitsyn ${ }^{3}$ \\ ${ }^{1}$ Space Research Institute (IKI), Moscow, Russia \\ ${ }^{2}$ Institute of Radio Astronomy of Academy of Sciences of Ukraine, Kharkov, Ukraine \\ ${ }^{3}$ A. M. Obukhov Institute of Atmospheric Physics, Moscow, Russia
}

Received: 28 July 2007 - Revised: 3 March 2009 - Accepted: 11 March 2009 - Published: 26 March 2009

\begin{abstract}
It is commonly accepted, that cloud formation is caused by the humidity flux directed from the warm bottom atmospheric layers towards the cold dry heights, and the transportation mechanism in stable stratification is due to development of so-called turbulent boundary layer. The transportation of vapor can be described by buoyancy profile, and requires two significant characteristics of the atmosphere. The first is the heat and water vapor fluxes from the underlying surface which has been investigated by Smith (1988). The second is the temperature profile in the atmosphere, which is usually approximated and parameterized in various ways, because the exact solution is complicated and difficult to use. In this paper we construct a theory of three-component gas mixture, containing air, vapor, and water droplets. This model can be applied for the internal cloud region. Later we use buoyancy to investigate the dynamics of cloud formation, taking into account condensation of the water vapor inside the cloud. The obtained results suggest a typical time of $10 \mathrm{~h}$ required for development of intense cloud layer over a sea surface.
\end{abstract}

\section{Introduction}

Troposphere is the lowest atmospheric layer up to approximately $15 \mathrm{~km}$ height, which is responsible for climate formation, which in turn affects all sides of human life. This part of the atmosphere contains almost all atmospheric water vapor and features intensive dynamic motion of the air. In spite of low relative pressure of the vapor in the air, it is one of the most important components due to its condensation ability. The heat released during phase transition of vapor is

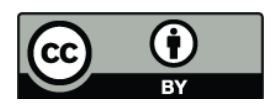

Correspondence to: P. B. Rutkevich (peter@d902.iki.rssi.ru) the main source of energy for variety of processes and atmospheric effects.

Since the relative densities of the main atmospheric gases $\left(\mathrm{N}_{2}, \mathrm{O}_{2}, \mathrm{Ar}, \mathrm{CO}_{2}\right.$, and some others) are invariable within the troposphere, the dry component of the air is usually taken as a gas with atomic mass 29 a.m.u. Additional vapor component in the air changes the average density (due to smaller atomic weight 18 a.m.u.) and also changes the adiabatic profile due to its triatomic molecules, which have the heat capacity at constant volume $c_{V w}=3 R$ instead of $c_{V d}=5 R / 2$ for two-atomic gas, where $R=8.31 \mathrm{~J} /(\mathrm{mol} \cdot \mathrm{K})$ is the absolute gas constant, and indexes $w$ and $d$ stand for water vapor and $d r y$ air components.

Temperature gradient, which is the main parameter in the atmosphere science, can vary in real atmosphere between $\approx-6 \mathrm{~K} / \mathrm{km}$ (for saturated water vapor adiabatic profile) to $\approx-10 \mathrm{~K} / \mathrm{km}$ (for dry adiabatic profile). The exact solution of a two-component gas mixture requires solution of a set of ordinary differential equations with boundary conditions, and is rarely used. Instead there exist several ways for parameterization of atmospheric parameter profiles, most of them use the condition of small water vapor pressure. Commonly used quantities are virtual temperature $T_{v}$, i.e. the temperature dry air would have to yield the same density as moist air, and density temperature $T_{\rho}$, which has a similar meaning as $T_{v}$ but used for the air with condensed water or crystalline ice (Emanuel, 1994).

Such parameterizations and numerical approximations are mainly used because of their simplicity, however, being applied to the internal cloud region they hide the physics of the two-component (or even three-component for condensed water inside the cloud) gas mixture. In this work we use thermodynamics of a three-component gas mixture with phase transitions, and apply this model for dynamics of cloud formation and vapor transport in presence of gravity.

The next process of our interest is vertical transportation of water vapor and heat from the surface. The vapor transport

Published by Copernicus Publications on behalf of the European Geosciences Union. 
significantly depends on the state of the atmosphere, i.e. convection or inversion. Given the initial air distribution is stable (which often happens when the cold dry air blows over a warm sea) the convective layer grows gradually from the surface. A powerful technique to describe this kind of convection is based on buoyancy, as it was shows by Zilitinkevich (1987). In the buoyancy approximation, the fluid is divided into initial (usually cold) upper part, and warm convective layer below. Significant temperature drop between these to parts leads to intensive local stirring on the top of the layer, which is the main mechanism of the layer growth. This model requires continuous flux of water vapor and heat from the underlying surface.

The buoyancy model can describe the boundary layer growth below the dew point, however, phase transitions inside the cloud make the model more complicated. Such model in the cloud requires thermodynamical description, where the temperature profile and the water vapor content affect each other. Using the thermodynamical model of the cloud derived in the first part of this paper, we formulate the buoyancy model for cloud with condensation. This model describes dynamics of the upper cloud edge growth.

The paper is organized as follows: in Sect. 2 we analytically determine the moisture dew point which corresponds to the lowest cloud boundary, and above this point the existing buoyancy model is not applicable. Section 3 presents thermodynamics of a three-component gas mixture with one gas being in equilibrium with its liquid phase. Here we derive moist thermodynamics profiles to be used in Sect. 4. Section 4 introduces the buoyancy model with vapor condensation and investigates dynamics of the cloud formation, i.e. the growth of the upper cloud boundary with time. Finally, the last section will summarize the results and briefly describe limitations of the model.

\section{Position of the cloud base}

Starting point of a cloud formation (i.e. the position of the cloud base) corresponds to the condensation level of vapor in the atmosphere. For a general case of a two-component gas this condition is often solved numerically or by means of various approximations (see, for example, Bolton, 1980).

However, an analytical expression can also be obtained. Indeed, in a good approximation one can use adiabatic profile for dry air

$T(z)=T_{0}\left(1-g z / c_{P} T_{0}\right)$

and the corresponding pressure profile $P(z)=P_{0}\left(1-g z / c_{P} T_{0}\right)^{c_{P} / R}$ Emanuel (1994) (here $c_{P}$ is the heat capacity of the two-component mixture). The axis $z$ is directed upwards with zero value at the sea surface. We consider the water vapor to be ideal gas with the state equation:

$E(z)=q(z) \rho_{d} R_{w} T$, where we use a new variable $q(z)=\rho_{w}(z) / \rho_{d}(z)$ for the vapor distribution. The relative density of the vapor below the cloud is constant $q(z)=r q_{0}=$ const, due to strong convective stirring, and the vapor pressure distribution below the cloud can be written as:

$E(z)=r q_{0} \rho(z) R_{w} T(z)=r q_{0} \rho_{0} R_{w}\left(T_{0}-\frac{g z}{c_{P}}\right)^{\frac{c_{P}}{R}}$,

where $r$ stands for the relative humidity at the sea surface level, and $q_{0}=q(0)$.

For any given temperature profile $T(z)$ one can find the saturation vapor pressure profile $E_{s}(z)$ using the ClapeyronClausius equation, or other parameterizations. The saturation pressure distribution based on the Clapeyron-Clausius equation can be expressed as

$E_{s}(z)=E_{s 0} \exp \left[\frac{L}{R_{w}}\left(\frac{1}{T_{0}}-\frac{1}{T(z)}\right)\right]$,

here $E_{s 0}$ is the saturated vapor pressure at the ocean surface, $L$ is the latent condensation heat, $R_{w}=R / \mu_{w}$ gas constant for water vapor, where $\mu_{w}$ is molar mass of water, $T_{0}$ temperature of the ocean surface, $T(z)$ is the temperature distribution.

Equating the expressions (4) and (3) one gets equation for the condensation height:

$r\left(1-\frac{g z}{c_{P} T_{0}}\right)^{\frac{c_{P}}{R}}=\exp \left[\frac{L}{R_{w}}\left(\frac{1}{T_{0}}-\frac{1}{T(z)}\right)\right]$,

where we have used the ideal gas law for the vapor $E_{s 0}=q_{0} \rho_{0} R_{w} T_{0}$. Equation (5) can be expanded into a series with respect to a small parameter $\varepsilon=1-T(z) / T_{0}=\frac{g z}{c_{P} T_{0}} \ll 1$ :

$\ln \left(r^{-1}\right)-\alpha \varepsilon+\left(\frac{c_{P}}{2 R}-\alpha\right) \varepsilon^{2}+o(\varepsilon)=0$,

where dimensionless parameter $\alpha=\frac{L}{R_{w} T_{0}}-\frac{c_{P}}{R}$, depends on the temperature at the sea surface. For example, numerical value of this parameter at $T_{0}=27^{\circ} \mathrm{C}$ is $\alpha=12.8$.

Solution of Eq. (6) in the simplest (linear) case is

$\varepsilon_{c}^{(1)}=\frac{\ln \left(r^{-1}\right)}{\alpha}$,

where subscript $c$ stands for the cloud lower edge. Keeping the terms up to $\varepsilon^{2}$ one gets a more accurate solution of Eq. (6):

$\varepsilon_{c}^{(2)}=\frac{R}{c_{P}} \frac{\alpha-\sqrt{\alpha^{2}+4 \alpha \ln (r)-2 \ln (r) c_{P} / R}}{1-2 \alpha R / c_{P}}$.

The dew point height (water vapor condensation level) $z_{c}$ is then determined as:

$z_{c}^{(1,2)}=z^{*}=\frac{c_{P} T_{0} \varepsilon_{c}^{(1,2)}}{g}$.

The corresponding temperature $T^{*}$, vapor density $q^{*}$ and other parameters can be found from the adiabatic profiles (1). 
The analytical expression (8) in assumption of dry adiabatic air distribution gives the result accurate within $2 \%$ of the previous results of Bolton (1980) and Emanuel (1994). Noteworthy, that Eqs. (6)-(8) are more generic, because they determine the condensation temperature, which may correspond to different height, depending on the actual temperature profile in the atmosphere.

\section{Moist air thermodynamics in the cloud}

To decribe a two-component gas mixture we use the following thermodynamic parameters: pressure $P$, dry air density $\rho$, temperature $T$, relative vapor density $q$, and vapor pressure $E$. Note, that unlike the under-cloud area, the relative vapor density $q$ inside the cloud varies with altitude due to the water condensation. Due to relatively low temperature in the cloud, one can safely assume $q \ll 1$ and $E \ll P$.

These variables are governed by the perfect gas laws for the dry air $P=\rho_{d} R_{d} T$ and the water vapor component $E=q \rho_{d} R_{w} T$. The vapor content becomes $q=R_{d} E / R_{w} P$, where we use the gas constant per unit mass $R_{d}=R / \mu_{d}$ for dry air ( $\mu_{d}$ is the molar mass of air), and the gas constant for vapor $R_{w}$ has been defined earlier. The Clapeyron-Clausius equation (4) in differential form becomes

$\frac{d E}{E}=\frac{L d T}{R_{w} T^{2}}$,

and differentials of other equations

$$
\begin{gathered}
\frac{d P}{P}=\frac{d \rho_{d}}{\rho_{d}}+\frac{d T}{T}, \quad \frac{d E}{E}=\frac{d q}{q}+\frac{d \rho_{d}}{\rho_{d}}+\frac{d T}{T}, \\
\frac{d q}{q}=\frac{d E}{E}-\frac{d P}{P} .
\end{gathered}
$$

The thermodynamic properties can be derived from the heat balance analysis. A portion of heat $\delta Q$ tranfered to a unit mass of moist air includes growth of its internal energy $d U=c_{V} d T$, the work of the gas $P d V$, and evaporation of vapor $L d q$ :

$\delta Q=T d S=c_{V} d T+P d V+L d q$,

where one can use $d V / V \approx d \rho_{d} / \rho_{d}$, due to small partial density of the vapor $\left(\rho_{w} \ll \rho_{d}\right)$.

Assuming in (12) an adiabatic profile $(d S=0)$, and eliminating the differentials $d T, d E$ and $d q$ from the set of Eqs. (11)-(12), gives the sound velocity in moist air:

$c^{2}(z)=\frac{\partial P}{\partial \rho}=R T \frac{1-\frac{L q}{T c_{P}}+\frac{L^{2} q}{c_{P} R_{w} T^{2}}}{\frac{c_{V}}{c_{P}}-\frac{L q}{c_{P} T}+\frac{L^{2} q}{c_{P} R_{w} T^{2}}}$.

Using Eqs. (10)-(11), Eq. (13) and the vertical pressure profile from stationary Navie-Stokes equation $d P=-g \rho_{d} d z$, one obtains the following set of nonliear equations for $T$ and $q$ :

$$
\begin{gathered}
\Gamma_{a}=\frac{1}{T} \frac{d T}{d z}=-\frac{g}{R_{d} T} \frac{\frac{R_{d}}{c_{P}}+\frac{L q}{c_{P} T}}{1+\frac{L^{2} q}{c_{P} R_{d} T^{2}}}, \\
\Gamma_{q}=\frac{1}{q} \frac{d q}{d z}=-\frac{g}{R_{d} T} \frac{\frac{R_{d}}{R_{w}} \frac{L q}{c_{P} T}-1}{1+\frac{L^{2} q}{c_{P} R_{d} T^{2}}} .
\end{gathered}
$$

These two equations can be solved either numerically, or analytically in certain approximations, as we will show below. Profiles of other thermodynamic parameters

$$
\begin{aligned}
& \Gamma_{P}=\frac{1}{P} \frac{d P}{d z}=-\frac{g}{R_{d} T}, \quad \Gamma_{\rho}=\frac{1}{\rho} \frac{d \rho}{d z}=-\frac{g}{c^{2}(z)}, \\
& \Gamma_{E}=\frac{1}{E} \frac{d E}{d z}=\frac{L}{R_{w} T} \Gamma_{a}
\end{aligned}
$$

can be straightforwardly obtained from the solutions of the system Eqs. (13)-(15).

Equations (14)-(17) are applicable for the internal cloud region $z \geq z_{c}$. We use asterisk for the vapor content and temperature values at the lower cloud edge: $q\left(z_{c}\right)=q^{*}$ and $T\left(z_{c}\right)=T^{*}$. Assuming that the vapor content changes slowly and substituting $q=q^{*}$ and $T=T^{*}$ in the RHS of Eq. (14) one can integrate it and obtain approximate solution for temperature

$T(z)=T^{*}-\frac{\frac{R_{d}}{c_{P}}+\frac{L q^{*}}{c_{P} T^{*}}}{1+\frac{L^{2} q^{*}}{c_{P} R_{w} T^{* 2}}} \frac{g z}{R_{d} T^{*}}$.

Similarly, substituting $T=T^{*}$ in the RHS of Eq. (15) after integration one obtains an implicit expression for $q(z)$ :

$\frac{L^{2}\left(q-q^{*}\right)}{c_{P} R_{w} T^{* 2}}+\ln \frac{q}{q^{*}}=-\frac{g z}{R_{d} T^{*}}\left(\frac{R_{d}}{R_{w}} \frac{L}{c_{P} T^{*}}-1\right)$.

Two terms in the LHS of Eq. (19) suggest that the cloud consists of two sub-layers with essentially different properties, as shown in Fig. 1. These sub-layers can be called as the lower cloud and the upper cloud. The lower cloud can be described using the first (linear) term in LHS of this equation together with the logarithm expanded to a linear approximation. In this approximation

$q(z)=q^{*}\left(1-\frac{\frac{R_{d}}{R_{w}} \frac{L}{c_{P} T^{*}}-1}{1+\frac{L^{2} q^{*}}{c_{P} R_{w} T^{* 2}}} \frac{g\left(z-z_{c}\right)}{R_{d} T^{*}}\right)$.

The height where the third term in the logarythm expansion is equal to the second term, and therefore the used expansion is not valid, determines the boundary between the cloud sublayers:

$z_{b}=z_{c}+\frac{R_{d} T^{*}}{g} \frac{1+\frac{L^{2} q^{*}}{c_{P} R_{w} T^{* 2}}}{\frac{R_{d}}{R_{W}} \frac{L}{R_{w} T^{*}}-1}$. 




Fig. 1. Vertical distributions of vapor mixing ratio $q(z)$ and temperature $T(z)$ in moist atmosphere. Solid line represents the numerical solution of Eq. (19), and dash and dot are linear (Eq. 20) and quadratic approximations, respectively. Dash-dot line shows the temperature profile in the cloud (Eq. 18), the average moist temperature profile $6^{\circ} \mathrm{C} / \mathrm{km}$ is shown for reference.

In the lower cloud $z_{c}<z<z_{b}$ the linear decrease of the vapor content is observed, while in the upper could it vanishes exponentially. In real atmosphere both $z_{c}$ and $z_{b}$ are within the atmosphere height.

It is worth to note that although Eqs. (14) and (15) were solved separately, the temperature profile (14) also changes its behavior at the same point $z_{b}$. Indeed, the temperature profile in the lower sub-layer is described following the moist adiabatic formula (18), while at the upper cloud layer the temperature profile is near the dry adiabatic formula $T(z) \approx T^{*}-g z / c_{P} T^{*}$. This result is obtained based on moist thermodynamics and is valid for any region with saturated moist air, and not only heavy cloudiness. In the upper atmosphere the temperature profile follows the dry-adiabatic profile.

\section{Cloud development due to evaporation from the sea surface}

Evaporation and heat flux from a warm ocean surface generate an unstable convective layer near the surface, which gradually penetrates into the stable atmosphere above. Such penetration is mainly regulated by the stirring effect on the top edge of the convective layer. It is convenient to introduce so called buoyancy for description of convective problems:

$b(z)=g \frac{\rho_{0}(z)-\rho(z)}{\rho_{0}(z)}$,

where $\rho_{0}(z)$ is the density profile in adiabatic atmosphere, $\rho(z)$ is the actual density profile, and the air density variation $\left(\rho_{0}-\rho\right)$ is subject to the temperature variation and variation of vapor content in the air.
Following Zilitinkevich (1987), the buoyancy transport equation for dry air can be written as:

$\frac{\partial b}{\partial t}=-\frac{\partial B(z)}{\partial z}$,

where $B$ is the vertical buoyancy flux, and it accounts both heat and vapor transport from the sea. Since the flux originates from the sea surface and the buoyancy value within the convective layer is constant (Smith, 1988), one can use in Eq. (23) a constant value of flux, equal to the buoyancy flux at the lowest level $B=B_{0}$. In application to the dry air, Eq. (23) can be solved using technique of Zilitinkevich (1987), which yields the layer thickness growth following the square root law $h=\sqrt{2 B_{0} t} / N$, where $N$ is the Brunt-Vaisala frequency.

However, above the condensation point $z_{c}$ the equation (23) is not complete anymore, and must account for the vapor condensation. The heat released inside the cloud produces additional source of buoyancy and therefore requires additional term in the RHS of the buoyancy balance equation:

$\frac{\partial b}{\partial t}=-\frac{\partial B}{\partial z}+\frac{g L}{T_{0} c_{P} T_{0}} \frac{d \rho_{l}(z)}{d t}$,

where $d \rho_{l}(z) / d t$ is the vapor amount (divided by the density of the dry air) condensed per unit time, per unit volume as a function of coordinate $z$, and $\rho_{l}\left(z<z_{c}\right)=0$. Later we will use vapor flux $\varphi(t)$ responsible for transport of the vapor amount $\rho_{l}$.

The partial differential equation (24) can be significantly simplified by proper parameterization of the buoyancy profile $b(z)$. Accounting intensive turbulent stirring inside the convective layer, the value of buoyancy is transforming towards a constant $b=b_{0}$ profile, while in the initial stable dry atmosphere the profile was following a linear function $b=N^{2} z$, where the frequency $N$ was introduced earlier. The buoyancy flux $B$ from the surface fills up the difference between the original linear profile and its final constant value $b_{0}$.

Following technique of Zilitinkevich (1987) and buoyancy parameterization we integrate Eq. (24) over $z$ from 0 to the top cloud edge $h$. The equation for the layer thickness $h$ becomes:

$\frac{d}{d t}\left(\frac{1}{2} N^{2} h^{2}-h \Delta b\right)=B_{0}(t)+\frac{g L}{T_{0} c_{P} \rho_{0}} \varphi_{0}(t)$,

where we assumed uniform condensation over the cloud thickness:

$\frac{d q}{d t}=\frac{\varphi(t)}{h}, \quad z_{c}<z<h$.

Fluxes $B_{0}=c_{0} g U \Delta T / T_{0}$ and $\varphi_{0}(t)=q_{0} \sqrt{B_{0}} /(N \sqrt{2 t})$ were chosen following Smith (1988).

Solution of Eq. (25) in the cloudy layer (that starts at the moment of cloud formation and upwards from point $z_{c}$ ) determines the cloud development:

$h=\frac{\sqrt{2}}{N} \sqrt{B_{0} t+\theta\left(t-t_{c}\right) \frac{\left(t-t_{c}\right) g L}{T_{0} c_{P} \rho_{0}} \varphi_{0}}, \quad t>t_{c}$, 


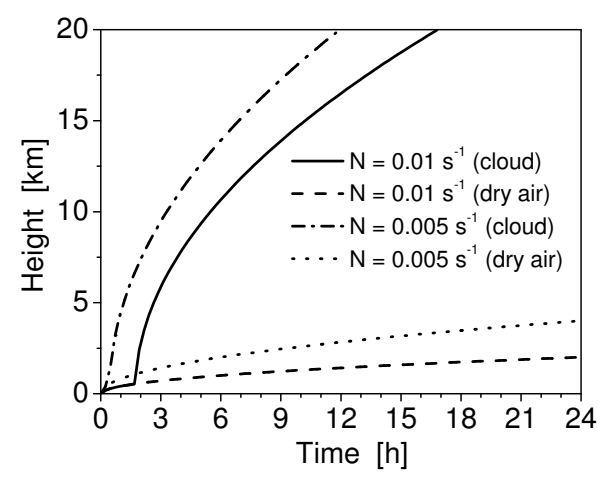

Fig. 2. Temporal development of the turbulent penetrative convective layer upper boundary in atmosphere over the ocean due to evaporation. Dash dot line is the layer development in dry atmosphere, solid line - the layer development in usual atmosphere with a cloud forming at some height.

where $\theta(x)$ is the Heaviside function equal to 1 for positive arguments, and 0 for negative $x$. Formation of the cloud starts at $t=t_{c}$. Equation (27) describes formation of convective layer both in the sub-cloud and in the cloud, as one can see in Fig. 2. The released heat in the cloud results in remarkable increase of the buoyancy flux above the condensation point. Such behaviour of buoyancy flux was observed by Bretherton (1997), and it is responsible for the cloud formation.

\section{Discussion and conclusions}

Depending on the thermodynamic profiles, atmosphere can be characterized as either stable, neutral, or unstable. The fastest mechanism of vapor transportation is through development of convective layer, which can appear in any of the above situations. The most challenging question is growth of convective layer and vapor transportation in stable stratification, since in initially unstable atmosphere the upwards flux is fast and intensive.

Development of convective layer in initially stable air is impossible without accounting for low-scale stirring, which gradually involves higher atmosphere levels. Such formation cannot be explained based on linear Navier-Stokes equations. However, using inequality $t_{\text {turb }} \ll t_{\text {con }}$, where $t_{\text {turb }}$ is the time of turbulent pulsations responsible for the stirring and $t_{\text {con }}$ is the time of formation of the convective layer, the process can be described in a linear approximation Eq. (25) based on buoyancy formalism, and even can be transformed from partial to ordinary differential equation. Buoyancy approximation requires a guess of vertical distribution of the neutral profiles, thus thermodynamical model of the cloud is needed.

Stable atmosphere has natural division into surface layer (responsible for evaporation), dry air with unsaturated vapor component, and the cloud layer containing air with saturated vapor. The dew point height as the function of relative humidity in the sea surface of subcloud layer can be found, and profiles of thermodynamic parameters in three-component gas mixture (dry air, vapor and liquid water) introduced.

According to the three-component thermodynamic model, the cloud consists of two significantly different sub-layers (Fig. 1), in particular, the lower sub-cloud with significant vapor content, and upper cloud where the vapor content vanishes due to low temperature (though it still remains saturated). The boundary in heavy cloud system that divides linear and exponential decrease of the vapor density depends on other thermodynamic parameters. These results are in accordance with measured temperature profiles in the tropic clouds.

Formation of convective layer is a dynamic process, since it appears due to evaporation from relatively warm ocean surface. The under-cloud area can be described following the model of Zilitinkevich (1987), while above the dew point the effective buoyancy flux from the sea surface $B_{0}$ gains additional term due to vapor condensation and released heat, and above the lower cloud boundary the convective layer expansion occurs more rapidly than in the dry air below. Therefore, the second term in the RHS of Eq. (24) can be addressed as latent buoyancy flux, which is released only above condensation level $z_{c}$.

As a result, the cloud growth rate suddenly increases when the convective layer reaches the condensation point, and formation time of the clouds can be as short as a half a day (Fig. 2). For more intensive evaporation this process will be even faster. In the proposed model the cloud growth is limited by the temperature inversion in the upper atmosphere, which is located at approximately $15 \mathrm{~km}$ in the tropics, and the model does not account for atmosphere heating due to absorption of the solar radiation.

Acknowledgements. This work was supported by the RFBR grant no. 09-05-00374-a.

Edited by: U. Harlander

Reviewed by: two anonymous referees

\section{References}

Bolton, D.: The computation of equivalent potential temperature, Mon. Weather Rev., 108, 1046-1053, 1980.

Bretherton, C. S.: Convection in stratocumulus-topped atmospheric boundary layers, in: The physics and Parameterization of Moist Atmospheric convection, edited by: Smith, R. K., Kluwer Acad. Pub., 127-142, 1997.

Emanuel, K. A. : Atmospheric convection, Oxford University Press, Oxford, 1994.

Smith, S. D.: Coefficients for Sea Surface Wind Stress, Heat Flux, and Wind Profiles as a Function of Wind Speed and Temperature, J. Geophys. Res., 93(C12), 15467-15472, 1988.

Zilitinkevich, S. S.: Theoretical model of turbulent penetrative convection, Izvestia AN SSSR, FAO, 23, 6, 593-610, 1987. 